\title{
Treatment strategies and outcome of pediatric cerebral cavernomas
}

\author{
George A. Alexiou $\cdot$ Neofytos Prodromou
}

Received: 21 October 2009/ Accepted: 22 June 2010/Published online: 2 July 2010

(C) Springer-Verlag 2010

\section{Dear Editor,}

We read with great interest the recent article by Consales et al. concerning the treatment and outcome of children with cerebral cavernomas. The authors prospectively studied 32 children with brain cavernomas. A great proportion of cavernomas (65.6\%) had macrohemorrhage, whereas $15.6 \%$ presented only with seizures. Surgical excision was performed in 28 patients and resulted in favorable long-term outcome and seizure control. The authors concluded that there is a high risk of macrohemorrhage in children with cavernomas and surgery should be performed in most surgically accessible cavernomas [1].

We retrospectively studied only the cerebral cavernomas that were treated surgically in our institute over the last 14 years. We identified 16 cases of pediatric cavernomas. The parietal lobe was the most common site of occurrence, whereas only two cavernomas were infratentorial. In our series, there were only 3 children with macrohemorrhage, whereas 14 children presented with seizures. Among them, 8 children had seizures that were controlled by medication, 2 cases were refractory to anticonvulsants and the remaining 4 patients were operated shortly after the first few seizures. In all cases, gross total resection was performed that included the surrounding gliotic and hemosiderin-stained brain parenchyma. After a mean follow-up period of 5.9 years, all patients were in good condition. Concerning the seizure outcome, as assessed by the Engel

G. A. Alexiou - N. Prodromou

Department of Neurosurgery,

Children's Hospital “Agia Sofia”, Athens, Greece

G. A. Alexiou $(\square)$

Aetideon 52, Holargos, 11561 Attikis, Greece

e-mail: alexiougrg@yahoo.gr; alexiougr@gmail.com classification, $11(78 \%)$ patients were seizure-free (class I) and $3(22 \%)$ patients were in class II [2].

Removal of the surrounding gliotic parenchyma and hemosiderin ring is a matter of debate. Ferroli et al. [3] reported that $68 / 99$ patients that presented with epilepsy were completely seizure free after pure lesionectomy alone. On the contrary, Hammen et al. [4] reported that complete resection of hemosiderin fringe surrounding the cavernoma was correlated to less post-operative seizure frequency. Baumann et al. [5] also found that complete removal of the cavernoma's surrounding hemosiderinstained brain tissue improved epileptic outcome. In our study, we removed the surrounding gliotic and hemosiderin-stained brain parenchyma in all patients and observed a favorable result on seizure control. To conclude, symptomatic cavernomas should be treated surgically and total resection is associated with favorable results.

\section{References}

1. Consales A, Piatelli G, Ravegnani M et al (2010) Treatment and outcome of children with cerebral cavernomas: a survey on 32 patients. Neurol Sci 31:117-123

2. Alexiou GA, Mpairamidis E, Sfakianos G, Prodromou N (2009) Surgical management of brain cavernomas in children. Pediatr Neurosurg 45:375-378

3. Ferroli P, Casazza M, Marras C et al (2006) Cerebral cavernomas and seizures: a retrospective study on 163 patients who underwent pure lesionectomy. Neurol Sci 26:390-394

4. Hammen T, Romstöck J, Dörfler A et al (2007) Prediction of postoperative outcome with special respect to removal of hemosiderin fringe: a study in patients with cavernous haemangiomas associated with symptomatic epilepsy. Seizure 16:248-253

5. Baumann CR, Schuknecht B, Lo Russo G et al (2006) Seizure outcome after resection of cavernous malformations is better when surrounding hemosiderin-stained brain also is removed. Epilepsia 47:563-566 